University of Nebraska - Lincoln

DigitalCommons@University of Nebraska - Lincoln

\title{
Feasibility of motor-assisted elliptical to improve walking, fitness and balance following pediatric acquired brain injury: A case series
}

Judith M. Burnfield

Madonna Rehabilitation Hospital, jburnfield@madonna.org

Guilherme Manna Cesar

Madonna Rehabilitation Hospitals, g.cesar@unf.edu

Thad W. Buster

Madonna Rehabilitation Hospitals, tbuster@madonna.org

Follow this and additional works at: https://digitalcommons.unl.edu/mechengfacpub

Part of the Mechanics of Materials Commons, Nanoscience and Nanotechnology Commons, Other Engineering Science and Materials Commons, and the Other Mechanical Engineering Commons

Burnfield, Judith M.; Cesar, Guilherme Manna; and Buster, Thad W., "Feasibility of motor-assisted elliptical to improve walking, fitness and balance following pediatric acquired brain injury: A case series" (2021). Mechanical \& Materials Engineering Faculty Publications. 604.

https://digitalcommons.unl.edu/mechengfacpub/604

This Article is brought to you for free and open access by the Mechanical \& Materials Engineering, Department of at DigitalCommons@University of Nebraska - Lincoln. It has been accepted for inclusion in Mechanical \& Materials Engineering Faculty Publications by an authorized administrator of DigitalCommons@University of Nebraska Lincoln. 


\title{
Feasibility of motor-assisted elliptical to improve walking, fitness and balance following pediatric acquired brain injury: A case series
}

\author{
Judith M. Burnfield, Guilherme M. Cesar, and Thad W. Buster
}

\author{
Institute for Rehabilitation Science and Engineering, \\ Madonna Rehabilitation Hospitals, Lincoln, NE, USA \\ Corresponding author - Judith M. Burnfield, PhD, PT, Institute for Rehabilitation Science and \\ Engineering, Madonna Rehabilitation Hospitals, 5401 South Street, Lincoln, NE, 68506, \\ USA; tel 402-413-4505; fax 402-413-4512; email jburnfield@madonna.org
}

\begin{abstract}
.
Purpose: Walking, fitness, and balance deficits are common following acquired brain injury (ABI). This study assessed feasibility, acceptability, and usefulness of a modified motor-assisted elliptical (ICARE) in addressing walking, fitness, and balance deficits in children with chronic ABIs.

Methods: Three children ( $>5$ years post-ABI) completed 24 ICARE exercise sessions (exercise time, speed, and time overriding motor-assistance gradually increased) to promote mass repetition of gait-like movements and challenge cardiorespiratory fitness. Parents' and children's perceptions of ICARE's safety, comfort, workout, and usability were assessed. Cardiovascular response, gait and balance outcomes were assessed.

Results: No adverse events occurred. Parent's Visual Analogue Scale (VAS) scores of perceived device safety (range 80-99), workout (range 99-100), and usability (range 75-100) were high, while comfort were 76-80 given commercial harness fit and arm support. Children's VAS scores all exceeded 89. Comfortable walking velocity, 2-Minute Walk Test, fitness, and Pediatric Balance Scale scores improved
\end{abstract}

\footnotetext{
Published in Journal of Pediatric Rehabilitation Medicine: An Interdisciplinary Approach

Throughout the Lifespan (2021)

DOI:10.3233/PRM-200717

Copyright (C) 2021 IOS Press. Used by permission.
} 
post-training, with many outcomes surpassing established minimal clinically important differences.

Conclusion: Following engagement in moderate- to vigorous-intensity exercise promoting repetitive step-like movements on a specially adapted motor-assisted elliptical, three children with chronic ABI demonstrated improvements in walking, fitness and balance. Future research in community-based environments with a larger cohort of children with $\mathrm{ABI}$ is needed.

Keywords: Children, brain injury, gait training, balance, fitness, technology, elliptical

\section{Introduction}

Not infrequently, children who survive a moderate or severe acquired brain injury (ABI) require extended rehabilitation and community-based interventions to address long-term challenges with walking in addition to lingering deficits in fitness and balance control. Intensive, task-specific training is believed to be critical for promoting functional recovery and lasting neuroplastic changes in gait following a neurologic injury $[1,2]$. Treatment approaches used during formal inpatient rehabilitation aren't always easily integrated into community-based programs as labor requirements (e.g., repetitively lifting a paretic lower extremity during partial body weight support treadmill training) and technology expense (e.g., robotics) may exceed the budget constraints of smaller clinics, medical fitness and school settings [3]. Further, the residual motor fitness [4] and balance deficits [5] that persist in many children postABI can make sustained stepping at recommended intensities difficult when navigating community environments or using traditional exercise devices (e.g., treadmills). Collectively, these challenges can contribute to a pat tern of persistent inactivity, functional decline and negative health sequelae. Identification of practical approaches for addressing persisting walking and deconditioning deficits in children with chronic ABI would be expected to improve not only function, cardiorespiratory fitness, and long-term health [6], but also conceivably cognition and neuroplasticity critical for learning [7].

Recently, a motor-assisted elliptical (ICARE) used to address walking, fitness, and balance needs of adults with physical disabilities was adapted to address rehabilitation needs of children (Fig. 1) [8-13]. The ICARE enables practice of gait-like movements at speeds up to $65 \mathrm{cy}-$ cles per minute (CPM) without requiring a clinician's physical assistance to manually lift and advance legs as might be required during body 


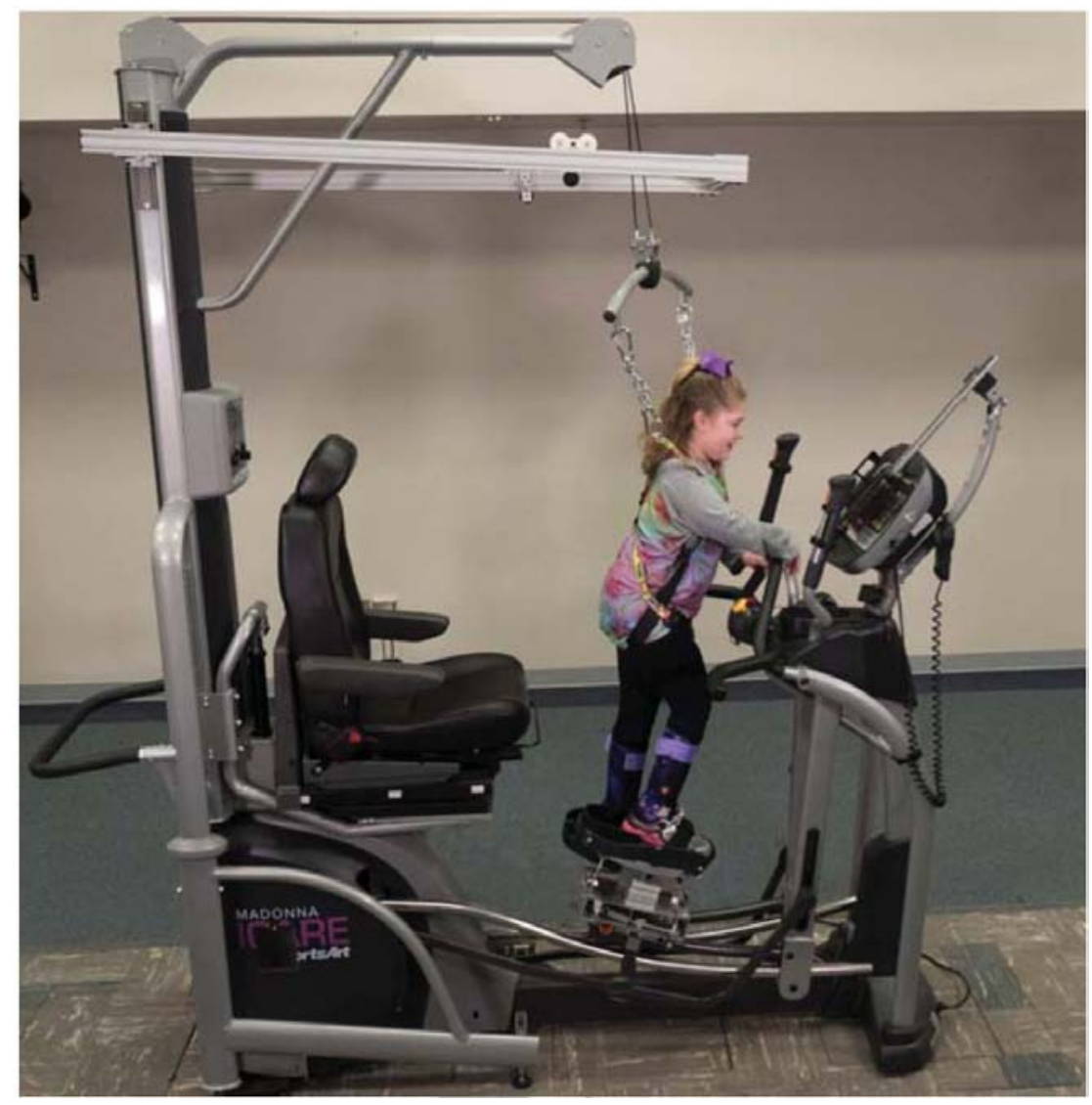

Fig. 1. Child 1 using ICARE. Note pedal elevation that allowed participant to use the stationary handles comfortably and to view and interact with the console. Consent to use photograph was obtained from the parent.

weight-supported treadmill training or overground gait approaches [8, $10,14]$. The device integrates three modes of motor assistance. During Active Assist training, an adjustable motor-drive system provides an assistive force that helps individuals with deconditioning sustain training at a pre-determined speed up to $65 \mathrm{CPM}$. To increase the challenge, brief bursts of Active Assist Plus training are often integrated within a session. During these brief bursts, users are encouraged to cycle at least $1 \mathrm{CPM}$ faster than the programmed training speed (feedback is provided on the device's console), causing the motor to disengage and no longer assist the cycling movement. Once the user slows to the programmed training speed (e.g., due to fatigue or the burst duration was achieved), the motor smoothly re-engages to support cycling at the pre-determined rate. Faster training speeds and overriding the motor's assistance incrementally increase the challenge to leg muscles and the heart $[9,10,15]$. The 
final training mode, Resistive, integrates 20 levels of resistance, characteristic of a traditional elliptical. Beyond being more affordable than many commercially available robotic gait training devices, the variety of motor assistance levels broadens ICARE's applicability and potentially reduces perceived stigma associated with training on a device solely designed for individuals with special health care needs and chronic conditions [8]. To address the needs of younger, often smaller stature users, step length adjusts to as short as $19 \mathrm{~cm}$ and the pedals can elevate so children can interact with the console. For those who are taller, step length can be increased to $71 \mathrm{~cm}$ and the pedals can be lowered incrementally. Multiple handholds and an external body weight support system allow children with weakness and balance challenges the support required to train without a clinician's physical assistance. Modifications to the ICARE for the pediatric population were driven, in part, by input from pediatric therapists in school, rehabilitation, and private clinics, as well as formal feedback provided by children with special healthcare needs and their caregivers [8].

This study's purpose was to assess feasibility, acceptability and usefulness of ICARE for addressing walking, balance, and fitness deficits in community- dwelling children with ABI. Given the novelty of the technology for pediatric ABI, we sought to understand 1) children's and parent's perceptions of the child's safety, comfort, and workout while using ICARE; 2) whether ICARE's parameters could be used to simultaneously promote high repetition practice of gait-like movements (> 1000 cycles/ session) and cardiorespiratory challenge; and 3) if walking, fitness, and balance would improve following participation. A secondary purpose of this study was to collect pilot data to guide sample size estimates for future intervention work.

\section{Methods}

\subsection{Participants}

Parental informed consent and informed assent from three children with ABI were obtained using procedures approved by the Institutional Review Board of Madonna Rehabilitation Hospitals.

Child 1 (8 years old, $1.32 \mathrm{~m}, 40.7 \mathrm{~kg}$ ), 7 years post a severe head trauma requiring ventriculostomy and 13-day sedation had persistent 
challenges with upper extremity control, balance, gait, and falling (several times/week). She ambulated with minimal to moderate assistance (short distances at home and in the community) and used a wheelchair for longer distances. Medications included docosahexaenoic acid (DHA) for brain health. She received 15 minutes/month of physical and occupation therapy at school and wore glasses.

Child 2 ( 7 years old, $1.32 \mathrm{~m}, 24.0 \mathrm{~kg}$ ), $\sim 6$ years post two cardiac arrests (30 minutes each; subsequently sedated for 7 days) with resultant hypoxia, vision loss, developmental delay, cognitive impairment, and challenges with walking (used bilateral supramalleolar orthoses), balance and falls. She took angiotensin-converting enzyme inhibitor and alpha-2A adrenergic receptor agonist (high blood pressure/heart failure) and received speech therapy.

Child 3 (9 years old, $1.41 \mathrm{~m}, 40.6 \mathrm{~kg}$ ), $\sim 7$ years post an automobile accident with resultant head trauma and 15-day induced coma, had left hemiplegia and ambulated without assistive devices. She had balance challenges during gait and several tripping/falling incidents weekly. She received levothyroxine (hypothyroidism) and norditropin injections (growth-related condition).

\subsection{Intervention}

Participants were initially familiarized with the adapted motor-assisted elliptical (ICARE E872MA, SportsArt, Mukilteo, WA; Fig. 1). The device's foot print was 2.2 meters in length by 0.8 meters in width. Baseline step length was identified. Then, speed was increased gradually until heart rate (HR) approximated 70\% estimated maximum (HRmax) [16], corresponding with estimates of moderate-intensity training. Resulting step length and speed served as baseline training parameters.

When using the ICARE, if the user's force input is sufficient to surpass the motor's set speed, assistance dynamically disengages and the user is able to exercise at any speed, within their individual physical capacity, above the set speed. Previous work determined that the physical effort (i.e., muscle and cardiorespiratory demands) increases at higher speeds regardless of motor assistance and further increases were observed when overriding the motor's assistance $[9,10,15]$.

Twenty-four sessions were scheduled (targeting 3/week). Prior to each session, resting HR was recorded (Masimo Rad-5v Signal Extraction

pulse oximeters; Masimo Corp., Irvine, CA, USA). Initial sessions were 
purposely shorter and limited to moderate-intensity to allow for exercise acclimation [17]. Our protocol targeted a $\sim 5 \%$ ICARE speed increase every 4 sessions and $\sim 5 \%$ exercise duration increase every session. Brief one-minute bursts of higher intensity exercise were introduced during session three by having each child override motor- assistance at pre-determined intervals. The number of higher-intensity bouts was scheduled to increase every two sessions. Exercise HR was monitored continuously with the Polar H1 Heart Rate Pro Sensor (Polar Electro Oy, Kempele, Finland), integrated via Radio Frequency with the ICARE's console. Training parameters were adapted to participants' responses.

\subsection{Outcome measures}

Session performance parameters were recorded including total time using ICARE ( $\mathrm{min}$ ), time over- riding ICARE's motor-assistance (min), ICARE's weighted average speed [CPM; (speed $\mathrm{x}$ time)/total exercise time], and total strides completed. Resting HR (beats per minute, bpm), exercise HR (with motor-assistance), and HR immediately following bouts overriding ICARE motor-assistance were recorded. The accuracy of HR data collected from the ICARE console has been previously verified [18].

Before the first and again following the 24th session, children traversed a GAITRite walkway at self-selected comfortable and fast speeds. Three trials were averaged to determine mean walking speed (m/s), cadence (steps/min), and stride length ( $\mathrm{cm}$ ) for each speed before and following intervention. Children also performed the 2-Minute Walk Test (2MWT) [19], modified Timed Up and Go (mTUG) [20] and Pediatric Balance Scale [21].

Following 24 sessions, children and parents provided quantitative (Visual Analogue Scale, VAS) and qualitative feedback regarding their perceptions of safety, comfort, workout (intensity of exercise), and usability of ICARE [8]. For safety, parents selected between 0 ("not safe at all-my child would get injured if someone weren't here") and 100 ("very safe-my child could use the equipment without worrying about injury") on the VAS and described why. For comfort while exercising on ICARE, parents selected between 0 ("not comfortable at all") and 100 ("very comfortable") and described why. For workout, parents selected between 0 ("a lousy work out") and 100 ("a great workout") and 
described why. For usability, parents selected between 0 ("my child wouldn't want to use it-it's useless for him/her") and 100 ("my child would really want to use it-it's ideal for him/her") and described why. Finally, parents responded to "If this piece of equipment was avail able for your child to use in your home/school/therapy setting, would your child use it? Why?"

Sample size estimation for each variable of interest was performed with $\mathrm{G} *$ Power $[22,23]$. Effect sizes were also calculated with this software to guide future intervention work.

\section{Results}

Child 1 and 2 required 64 days $(2.4 \pm 0.9$ and $2.4 \pm 0.5$ days/week, respectively) to complete the 24 sessions, while Child 3 required 55 days (3.0 \pm 1.1 days/week). Family scheduling conflicts contributed to differences across participants.

\subsection{Changes in ICARE training capacity across sessions}

\section{(Figs. 2 and 3)}

Child 1 trained with the adapted pedal raised $23 \mathrm{~cm}$ from the traditional pedal height to allow interaction at eye-height with ICARE's console. During session one, she trained for 17 minutes with motor-assistance at a weighted average speed of 22 CPM, allowing for a total of 375 strides. Average exercise HR across session one's exercise bouts was 116 bpm. During week one, she achieved 51 minutes of moderate- intensity exercise, including 1 minute performed while overriding motor-assistance. During week two, she completed 80 minutes of ICARE exercise, with 3 minutes performed at a more vigorous-intensity while overriding motor-assistance. By session 13, total training time increased to 36 minutes (including 6 minutes overriding motor-assistance) and speed was faster (weighted average 34 CPM), thus allowing for a greater than 3 -fold increase in total strides $(1,241)$ compared to baseline despite average exercise HR (117 bpm) being similar. Average HR while overriding motor-assistance increased to $126 \mathrm{bpm}$. During the final three sessions, she completed 152 minutes of moderate- to vigorous-intensity exercise (32 minutes overriding motor-assistance). 

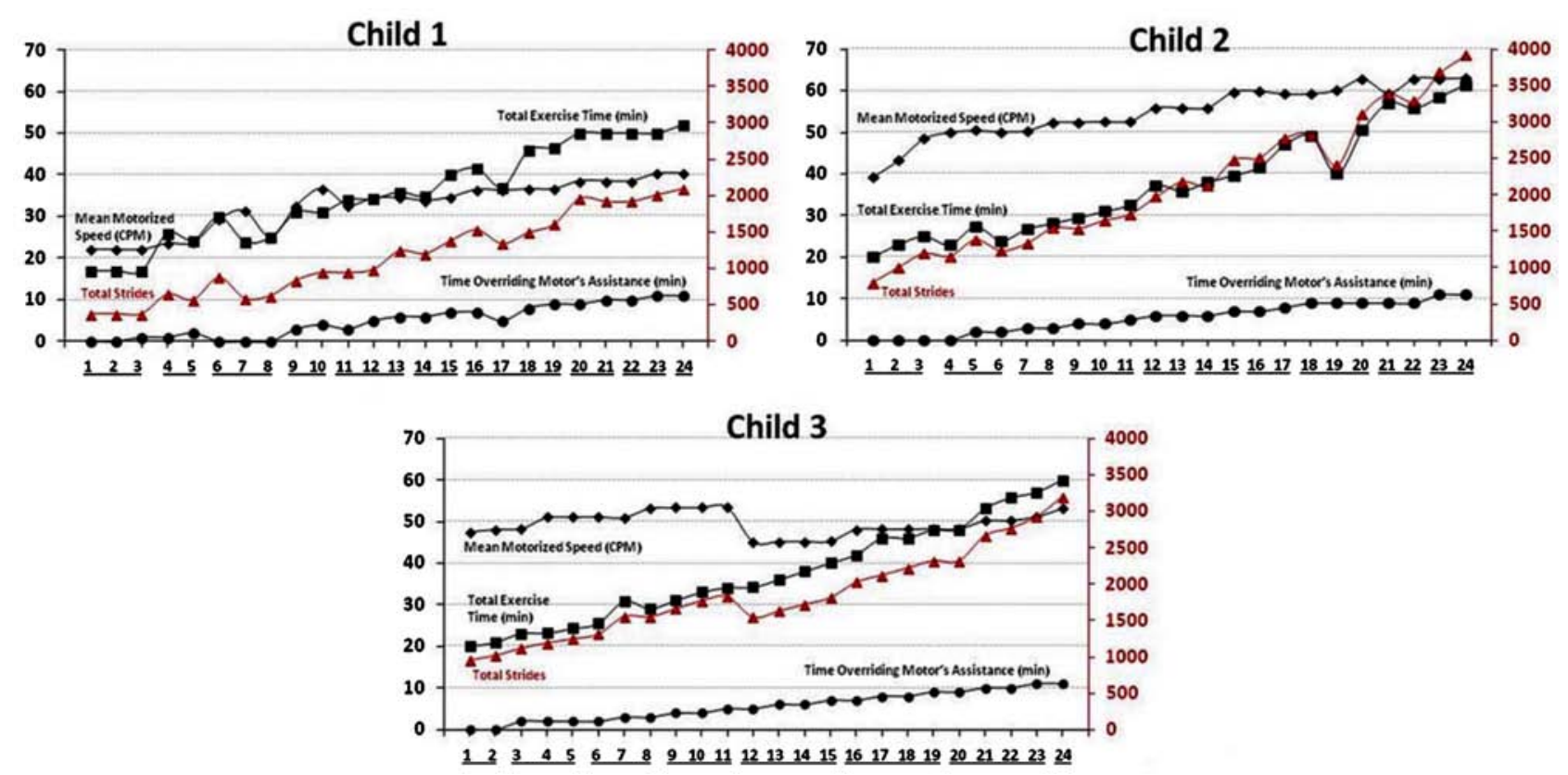

Fig. 2. Progression of ICARE training parameters across 24 sessions for each child. Mean motorized training speed (CPM; black diamonds), total exercise time (minutes; black squares), and time overriding the motor's assistance (minutes; black circles) are displayed with black lines and their respective values are provided on the left vertical axis. Total strides per session (red triangles) are displayed with red lines and the respective values are provided on the right vertical axis. Training sessions within the same calendar week are underlined on the $\mathrm{x}$-axis.

Her last session consisted of 52 minutes of moderate- to vigorous-intensity exercise (11 minutes overriding motor-assistance) performed at a weighted average speed of $40 \mathrm{CPM}$. The last session's total strides $(2,099)$ increased more than 5 -fold compared to base line. Average exercise HR (123 bpm) during these more challenging conditions was only slightly higher than the initial session and HR while overriding motor-assistance averaged $132 \mathrm{bpm}$. In total, Child 1 used ICARE for 844 minutes, overriding motor assistance for 118 minutes, and achieving 27,869 ICARE strides across 24 sessions.

Child 2 trained with the adapted pedal raised $13 \mathrm{~cm}$ from ICARE's standard pedal height. During session one, she trained for 20 minutes with motor-assistance at a weighted average speed of $39 \mathrm{CPM}$, allowing for a total of 785 strides. Average exercise HR across session one's exercise bouts was $116 \mathrm{bpm}$. During week one, she achieved 68 minutes 

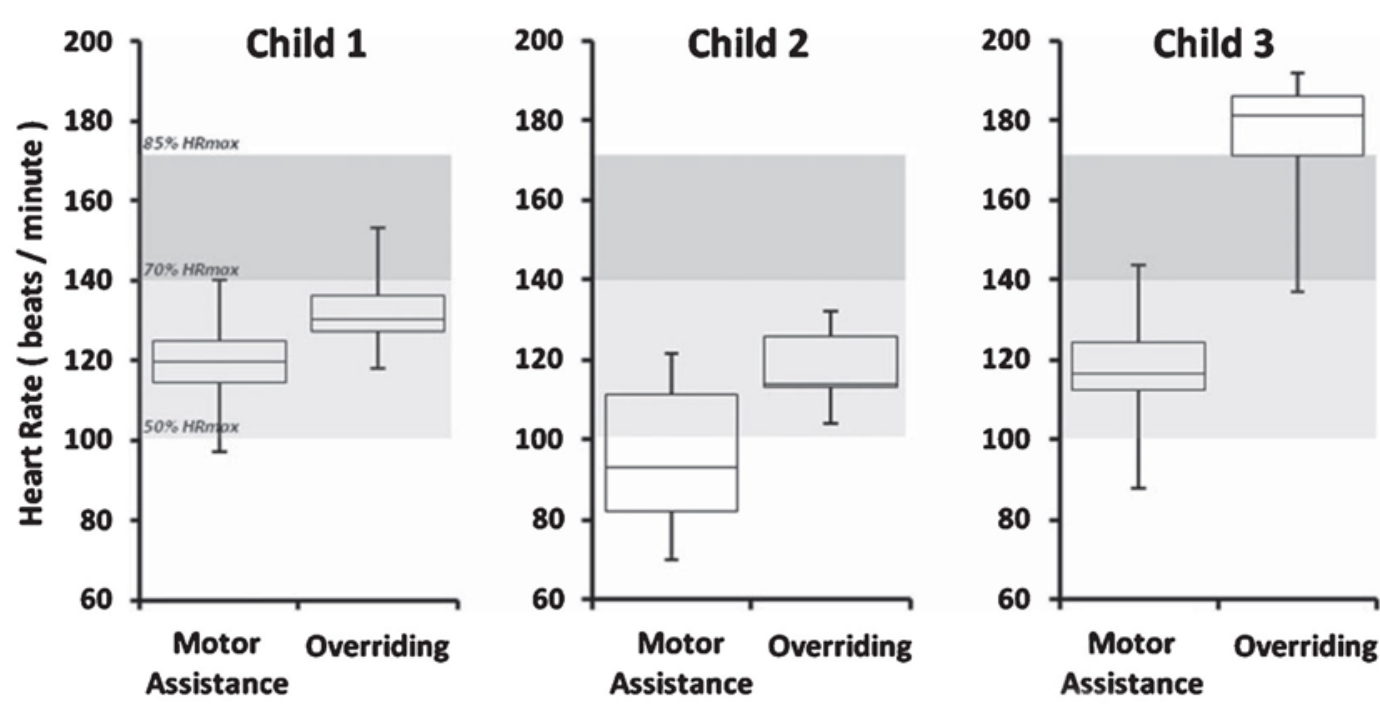

\begin{tabular}{lcccccc}
\hline & \multicolumn{2}{c}{ Child 1 } & \multicolumn{2}{c}{ Child 2 } & \multicolumn{2}{c}{ Child 3 } \\
\cline { 2 - 7 } & Resting HR & Exercise HR & Resting HR & Exercise HR & Resting HR & Exercise HR \\
\hline Initial Sessions & 103 & 118 & 101 & 103 & 93 & 129 \\
Later Sessions & 92 & 119 & 82 & 102 & 76 & 112 \\
\hline
\end{tabular}

Fig. 3. Average heart rate across all training sessions during ICARE exercise with the motor's assistance (left box plot for each child) and while overriding the motor's assistance (right box plot for each child). Expected age-appropriate heart rate ranges are displayed for moderate-intensity (light gray band) and vigorous-intensity (dark gray band) exercise. The midline within each box represents the median of the heart rate data, with the box representing $50 \%$ of the data points around the median and the additional top and bottom whiskers each representing the remainder $25 \%$ of the data. Resting heart rate prior to exercising and exercise heart rate with the motor's assistance are also provided as an average of the first three initial sessions and the last three sessions for each child.

of moderate-intensity exercise. During week two, she completed more than 74 minutes of ICARE exercise, with 4 minutes per-formed at a more vigorous intensity while overriding motor-assistance. By session 13, total training time increased to 36 minutes (6 minutes overriding motorassistance) and speed was faster (weighted average of $56 \mathrm{CPM}$ ), thus allowing for a nearly 3 -fold increase in total strides $(2,178)$ compared to baseline. This increase in activity was performed at a lower average exercise HR (94 bpm). Average HR while overriding motor-assistance was $121 \mathrm{bpm}$. During the final three sessions, she completed $176 \mathrm{~min}-$ utes of moderate- intensity exercise. By the final session, training was performed for 61 minutes (11 minutes overriding motor-assistance) at 
a faster speed (weighted average of 63 CPM). Total strides $(3,919)$ increased nearly 5- fold compared with baseline. Average exercise HR (104 bpm) was $10 \%$ lower than the initial session and HR while overriding motor-assistance averaged $129 \mathrm{bpm}$. In total, Child 2 used ICARE for 902 minutes, overriding motor-assistance for 130 minutes, and achieving 51,166 strides across the 24 sessions.

Child 3 trained with the adapted pedal raised $10 \mathrm{~cm}$ from ICARE's standard pedal height. During session one, she trained for 20 minutes with motor-assistance at an average speed of $48 \mathrm{CPM}$, allowing for a total of 953 strides. Average exercise HR across the first session's exercise bouts was $104 \mathrm{bpm}$. During week one, she achieved 64 minutes of moderate-intensity exercise and also overrode motor-assistance for 2 minutes to achieve vigorous-intensity exercise. During week two, which consisted of only two training sessions due to scheduling conflicts, she completed 48 minutes of ICARE exercise, with 4 minutes per formed at vigorous-intensity. During session 12 , her motor-assisted speed was lowered $19 \%$ from the previous session to facilitate success during 1-minute bouts overriding motor-assistance. By session 13, total training time increased to 36 minutes ( 6 minutes overriding motor-assistance) and incremental increases in speed were resumed (weighted average of 45 CPM), thus allowing for a 1.7 -fold increase in total strides $(1,624) \mathrm{com}$ pared with baseline. Step length was slightly longer $(48.0 \mathrm{~cm})$. Average HR while overriding motor-assistance was $178 \mathrm{bpm}$. During the final three training sessions, she completed 173 minutes of moderate- to vigorous-intensity exercise. By the final session, training was performed for 60 minutes (11 minutes overriding motor-assistance) at a faster speed (weighted average of 53 CPM). Total strides $(3,192)$ increased over 3-fold compared to baseline. Average exercise HR (109 bpm) was slightly lower than the initial session. Average HR while overriding motor-assistance was $165 \mathrm{bpm}$. In total, Child 3 used ICARE for $899 \mathrm{~min}$ utes, overriding motor-assistance for 134 minutes, and achieving 44,364 strides across the 24 sessions.

\subsection{Baseline to post-training changes in gait and balance (Table 1)}

During laboratory testing, Child 1 ambulated with minimal contact guard and without orthoses at base- line and post-training. Her comfortable 10MWT speed improved 23\% from baseline to post-training, 
Table 1 Walking and balance measures [mean (standard deviation) recorded across each child's three trials] before and following 24 ICARE training sessions for each child with an acquired brain injury

\begin{tabular}{|c|c|c|c|c|c|c|}
\hline & \multicolumn{2}{|c|}{ CHILD 1} & \multicolumn{2}{|c|}{ CHILD 2} & \multicolumn{2}{|c|}{ CHILD 3} \\
\hline & Baseline & Post & Baseline & Post & Baseline & Post \\
\hline \multicolumn{7}{|l|}{ Comfortable Pace } \\
\hline Speed $(\mathrm{m} / \mathrm{s})$ & $0.71(0.12)$ & $0.87(0.11)$ & $0.92(0.36)$ & $1.67(0.05)$ & $1.22(0.14)$ & $1.34(0.10)$ \\
\hline Stride Length $(\mathrm{cm})$ & $72(5)$ & $74(6)$ & $82(15)$ & $118(3)$ & $118(5)$ & $127(6)$ \\
\hline Cadence (steps/min) & $118(13)$ & $144(25)$ & $131(30)$ & $170(7)$ & $124(9)$ & $126(4)$ \\
\hline \multicolumn{7}{|l|}{ Fast Pace } \\
\hline Speed (m/s) & $1.15(0.07)$ & $1.04 *(0.02)$ & $2.00(0.24)$ & $2.24(0.05)$ & $1.72(0.05)$ & $1.80(0.04)$ \\
\hline Stride Length (cm) & $78(5)$ & $78(2)$ & $117(4)$ & $125(2)$ & $142(2)$ & $148(2)$ \\
\hline Cadence (steps/min) & $177(2)$ & $162(7)$ & $207(20)$ & $216(4)$ & $145(2)$ & $147(3)$ \\
\hline 2MWT $(\mathrm{m})$ & 89.9 & 91.4 & 124.1 & 176.8 & 170.4 & 192.0 \\
\hline mTUG (sec) & 12.2 & 12.4 & 7.7 & 7.0 & 6.3 & 5.1 \\
\hline \multicolumn{7}{|l|}{ Pediatric Balance Scale } \\
\hline Static & 15 & 16 & 15 & 14 & 24 & 24 \\
\hline Dynamic & 4 & 20 & 22 & 26 & 31 & 32 \\
\hline Total Score & 29 & 36 & 37 & 40 & 55 & 56 \\
\hline
\end{tabular}

* Post-assessment equipment challenges resulted in participant having to repeat walking trials multiple times.

due primarily to increased cadence $(\sim 22 \%)$ and to a lesser extent stride length (3\%). Her fast 10MWT speed was 10\% slower post-training compared to baseline, owing to an $8 \%$ shorter stride length. The need to repeat her post-intervention fast gait trials multiple times due to the timing gate accidentally triggering by the person contact guarding her may have been fatiguing. During the subsequent 2MWT, she walked 1.5 meters farther post-training compared to baseline. Her mTUG required 0.2 seconds $(<2 \%)$ longer to complete post-training, an increment that did not meet the clinical significance threshold. Her PBS score improved 24\%, surpassing the minimal detectable change (MDC90) and minimal clinically important difference (MCID) score for children with CP [24]. The largest increase in PBS emerged from a 43\% increase in dynamic items.

Child 2 did not require physical assistance or assistive devices to complete baseline and post-training assessments. Her comfortable 10MWT speed improved $82 \%$ from baseline to post-training. Increases in stride length (44\%) and to a lesser extent cadence (30\%) contributed to her faster comfortable speed. Her self-selected fast walking pace quickened $(12 \%)$ from baseline to post-training, owing to increases in both stride 
length (7\%) and cadence (4\%). Her 43\% longer 2MWT distance posttraining compared to baseline surpassed the MCID of $16.6 \mathrm{~m}$ for children with disabilities who walk independently [25]. Additionally, her posttraining mTUG was completed $8 \%$ faster than baseline, exceeding the MCID of 0.36 seconds for similarly aged children with CP and GMFCS II $[20,26]$. Her 3-point PBS score improvement surpassed the MDC90 of 1.59 for children with CP [24]. The largest increase in PBS emerged due to an $18 \%$ increase in dynamic items, surpassing the MCID range score of 2.23-2.92 [24].

Child 3 did not require physical assistance or assistive devices to complete baseline and post training assessments. Her comfortable 10MWT speed improved $10 \%$ from baseline to post-training due primarily to increased stride length (8\%) and to a lesser extent cadence (2\%). Her fast walking pace quickened slightly (5\%) from baseline to post training due to modest increases in stride length (4\%) and cadence (2\%). Her 13\% longer 2MWT distance post-training compared to baseline surpassed the MCID for children with disabilities who walk independently [24]. An 18\% reduction in time required to complete the mTUG post-training compared to baseline exceeded the MCID for similarly aged children with CP and GMFCS II nearly 3-fold [20, 26]. Her PBS score at baseline was 1 point away from the measure's maximum score, which she achieved post-training.

\subsection{Feedback on safety, comfort, workout, and usability (Table 2)}

Only limited feedback on perceptions of the ICARE were obtained from children (Table 2). Per the parent, Child 1 did not understand the VAS scoring for the Safety and Comfort despite providing verbal responses to the associated open-ended questions. Despite reporting not feeling safe when the researcher moved her paretic spastic upper extremity to different handholds, Child 1 indicated she would like to use the device if it were available at school and valued being able to use the Ipad while training. Child 2's visual, cognitive and speech deficits prevented her from completing the VAS and from answering the openended questions associated with each VAS category. Child 3 offered VAS scores ranging from 89 to 100 for Safety, Comfort, Workout and Usability and answered affirmatively that she would use the device if 
Table 2 Feedback from each child and parent, including Visual Analogue Scale Score and qualitative comments regarding perceived safety, comfort, workout quality, and usability of motor-assisted elliptical to address training goals

\section{SAFETY}

\begin{tabular}{lll}
\hline Child & Child VAS Score & Parent VAS Score \\
\hline 1 & NA & 90 \\
2 & Unable to respond & 80 \\
3 & 89 & 99
\end{tabular}

What factors contributed to you feeling you were (your child was) safe on the equipment?

\begin{tabular}{lll} 
Child Child Comments & Parent Comments \\
\hline 1 & That somebody was holding onto me. & $\begin{array}{r}\text { The harness, the leg braces, the seat, the off button and having } \\
\text { people around to help. } \\
\text { There are plenty of supports available for the child, so this } \\
\text { provides safety with the many features (i.e., foot straps and } \\
\text { hand supports). }\end{array}$ \\
$\begin{array}{lll}\text { I like the chair lift. The machine is a big piece of equipment and } \\
\text { could be a huge challenge for little kids to get in position. I } \\
\text { also liked the heart monitor. Some kids with disabilities do } \\
\text { have heart issues that do need to be monitored. }\end{array}$
\end{tabular}

What factors contributed to you feeling you were not (your child was not) safe on the equipment?

\begin{tabular}{|c|c|c|}
\hline Child & Child Comments & Parent Comments \\
\hline 1 & $\begin{array}{l}\text { When someone was switching holding } \\
\text { my hand. }\end{array}$ & $\begin{array}{l}\text { She isn't at the level to do most activities safely by herself. } \\
\text { I think that the ICARE is safe, and can be a safe activity for her } \\
\text { to get exercise on as long as someone is around. }\end{array}$ \\
\hline 2 & Unable to respond. & $\begin{array}{l}\text { Slightly concerned with safety on equipment as my child is easily } \\
\text { distracted, and does not always look forward while riding. } \\
\text { I would worry about an injury if she did not have someone } \\
\text { cuing her while riding as to hold on, look forward, etc. }\end{array}$ \\
\hline 3 & Not having body straps. & NA \\
\hline
\end{tabular}

\section{COMFORT}

\begin{tabular}{lll}
\hline Child & Child VAS Score & Parent VAS Score \\
\hline 1 & NA & 76 \\
2 & Unable to respond & 80 \\
3 & 100 & 80
\end{tabular}

What factors contributed to you (your child) feeling comfortable on the equipment?

\begin{tabular}{|c|c|c|}
\hline Child & Child Comments & Parent Comments \\
\hline 1 & I don't know. & $\begin{array}{l}\text { The stride length and the ability to raise and lower the pedals help } \\
\text { her to be able to use the ICARE easier. The chair gives her a } \\
\text { place to rest. The harness helped her feel stable. }\end{array}$ \\
\hline 2 & Unable to respond. & $\begin{array}{l}\text { She mimics an adult riding a similar machine*, so it looks as } \\
\text { comfortable as I would expect to see. [Note: machine* being } \\
\text { referenced was ICARE device developed for adults and children } \\
\text { of taller stature.] }\end{array}$ \\
\hline 3 & The handles. & $\begin{array}{l}\text { The fact that she did not have to step up into position on the } \\
\text { machine. The chair was a huge benefit. She did use the other } \\
\text { machine* a couple times and watching her get on and off was } \\
\text { a challenge for her. She was unsure or uneasy with doing so. } \\
\text { She struggles with balance sometimes so I know she was trying } \\
\text { not to fall while getting on or off. }\end{array}$ \\
\hline
\end{tabular}


Table 2 (continued)

What factors contributed to you (your child) feeling uncomfortable on the equipment?

\begin{tabular}{|c|c|c|}
\hline Child & Child Comments & Parent Comments \\
\hline 1 & $\begin{array}{l}\text { When someone was switching with } \\
\text { 'holding paretic limb.' [Note: researcher } \\
\text { inserted own phrasing when } \\
\text { transcribing child's gesture and limb } \\
\text { reference.] }\end{array}$ & $\begin{array}{l}\text { The harness caused a decent amount of problems for her. } \\
\text { It would have been nice to have a place for her to put her } \\
\text { hand/arm that doesn't work. }\end{array}$ \\
\hline 2 & Unable to respond. & $\begin{array}{l}\text { My child is probably not always consistent in her pushing abilities } \\
\text { on the machine, which could cause her discomfort. However, } \\
\text { the machine keeps her at a set pace if she slows, so this may or } \\
\text { may not be a factor. }\end{array}$ \\
\hline 3 & No response provided. & $\begin{array}{l}\text { I noticed [my daughter] a lot of times trying to use the handle } \\
\text { bars and looking very uncomfortable while doing so. She } \\
\text { would move her hands around a lot trying to find a more } \\
\text { comfortable position for her hands. }\end{array}$ \\
\hline
\end{tabular}

WORKOUT

\begin{tabular}{lll}
\hline Child & Child VAS Score & Parent VAS Score \\
\hline 1 & 100 & 100 \\
2 & Unable to respond & 100 \\
3 & 93 & 99
\end{tabular}

What factors contributed to your perceptions of how good of a "workout" you (your child) could achieve on the equipment?

\begin{tabular}{lll} 
Child Child Comments & Parent Comments \\
\hline 1 & The Ipad. & Any workout on this equipment is a good workout. This is the \\
& most amount of exercise she has had over a long period of \\
& time in her entire life. I feel that this is easier on the joints and \\
& helps her body move easier than when she is walking. I loved \\
& that we could get her heart rate up to a decent level. I loved \\
& the ability to adjust the bodyweight support system based on \\
& how she was feeling. I also liked that she could sit and take a \\
& break when she needed to. \\
& She enjoyed riding the equipment, and had a burst of energy \\
& once completed. She also slept very well after the sessions. \\
& If she was sweating after she was done, she would say her legs \\
& felt weak and she would be more tired on the days she had \\
& workouts.
\end{tabular}

What factors contributed to your perceptions of how bad of a "workout" you (your child) could achieve on the equipment?

\begin{tabular}{lll} 
Child & Child Comments & Parent Comments \\
\hline 1 & When Ipad was dead. & I think [my daughter] could have had a better workout if \\
& she would have had a better harness. I felt like it was \\
uncomfortable and got in her way at times. I also think if she & would have a better place to put her right hand (the one that \\
& doesn't move well).
\end{tabular}


Table 2 (continued)

\begin{tabular}{|c|c|c|}
\hline Child & Child VAS Score & Parent VAS Score \\
\hline 1 & 100 & 100 \\
\hline 2 & Unable to respond & 100 \\
\hline 3 & 89 & 75 \\
\hline \multicolumn{3}{|c|}{$\begin{array}{l}\text { If this piece of equipment was available for you (your child) to use in your home/school/therapy setting, would you } \\
\text { (your child) use it? }\end{array}$} \\
\hline Child & Child Comments & Parent Comments \\
\hline 1 & Would like to use it at school; Yes. & $\begin{array}{l}\text { Yes. Because it is the best option for safe exercise for her and } \\
\text { there is nothing else out there that she can use as effectively } \\
\text { for exercise as the ICARE. }\end{array}$ \\
\hline 2 & Unable to respond. & $\begin{array}{l}\text { Yes. This is exactly a piece of equipment that would serve my child } \\
\text { well. I would just not leave her unattended while riding. }\end{array}$ \\
\hline 3 & Yes. & $\begin{array}{l}\text { Yes. It gives an all around workout. It is a great piece of } \\
\text { equipment. }\end{array}$ \\
\hline
\end{tabular}

available. She appreciated that her feet were secured, the device integrated handles, and that overriding the motor's assistance at a faster speed provided a good workout.

Overall, parents exhibited a positive perception about ICARE and their child's exercise experience (Table 2). Two areas identified by parents for further development included the need for additional arm supports/handlebars to help those with limited upper extremity function engage their arm(s) and revisions to the commercial body harness (not an ICARE product) given comfort concerns.

\subsection{Sample size estimates (Table 3)}

The estimated sample size for each variable is dis- played in Table 3. While assessments for comfortable walking pace, $2 \mathrm{MWT}$, and the dynamic component of PBS provided a sample size range from 7 to 28 individuals, other evaluations rendered much larger sample sizes likely due to the smaller observed effect size. 
Table 3 Post hoc sample size calculations and effect size estimates derived from data recorded for three participants with acquired brain injuries

\begin{tabular}{|c|c|c|}
\hline & $\begin{array}{l}\text { Sample Size } \\
\text { (0.80 Power) }\end{array}$ & $\begin{array}{l}\text { Effect } \\
\text { Size }\end{array}$ \\
\hline \multicolumn{3}{|l|}{ Comfortable Pace } \\
\hline Speed $(m / s)$ & 9 & 0.97 \\
\hline Stride Length (cm) & 20 & 0.59 \\
\hline Cadence (steps/min) & 7 & 1.13 \\
\hline \multicolumn{3}{|l|}{ Fast Pace } \\
\hline Speed $(\mathrm{m} / \mathrm{s})$ & 374 & 0.12 \\
\hline Stride Length $(\mathrm{cm})$ & 331 & 0.13 \\
\hline Cadence (steps/min) & 4033 & 0.04 \\
\hline 2MWT (m) & 25 & 0.52 \\
\hline mTUG (sec) & 242 & 0.16 \\
\hline \multicolumn{3}{|l|}{ Pediatric Balance Scale } \\
\hline Static & too many to calculate & $<0.00001$ \\
\hline Dynamic & 28 & 0.485 \\
\hline Total Score & 70 & 0.3 \\
\hline
\end{tabular}

\section{Discussion}

Long-term challenges with walking, fitness and balance are not uncommon following pediatric $\mathrm{ABI}$ and can affect children's ability to function effectively in home, school and social environments. Unfortunately, the resources (i.e., technology and therapists) needed to foster intensive, task-specific training are infrequently available in community environments. ICARE was originally developed so adults with physical disabilities and chronic conditions could work simultaneously on walking, fitness and balance goals during formal rehabilitation and following return to their communities and homes. The robotic nature of ICARE reduces need for clinicians or caregivers to physically assist children during training, although some children may still require assistance donning a harness. Additionally, the device can be used as a traditional elliptical (with 20 levels of resistance) by individuals without known disability thus broadening applicability in settings addressing the comprehensive needs of individuals of all abilities [8, 14,27]. While more expensive than traditional cardiorespiratory exercise devices (e.g., recumbent cycles, treadmills), ICARE is more affordable than most commercial robotic devices used for gait training. The current research advances upon 
a foundation of work focused on technology development and assessment with adults to help inform understanding of whether a modified ICARE can be used safely and effectively to address walking, fitness and balance goals in a small sample of children $(n=3)$ who have lingering challenges due to chronic ABIs.

\subsection{Feasibility of exercise protocol}

Consistent with the protocol's design, each child tolerated greater physiologic challenge (increased speed, more frequent bouts overriding motor assistance, and longer total training time) across the 24-session program and no adverse events occurred. Beyond creating the desired physiologic challenge, each child's parent reported generally feeling safe with children exercising on ICARE with Safety VAS scores ranging from 80/100 to 99/100. Parent 3 appreciated ICARE's integrated HR monitor for those with cardiac issues. Parent 2 emphasized someone should be present while children train on ICARE, particularly those who are easily distracted. Despite multiple safety features, we concur that guidance should be provided commensurate with a user's cognitive and physical needs.

Each parent perceived the device as valuable for their child's training as evidenced by Workout VAS scores ranging from 99/100 to 100/100 across the three participants and reported benefits spanning beyond function and fitness. One parent indicated her daughter "slept well" after training. The two children capable of responding to the questions and all three parents responded "yes" to "If this piece of equipment was available for you (or your child) to use in your home/school/therapy setting, would you (your child) use it?"

Two items were identified for improvement. As currently designed, ICARE allows hand placement on two static hand holds and a dynamic set of reciprocally moving handles. However, these handles were challenging for two children to grasp given upper extremity weakness and spasticity. Work is underway to develop adjustable reciprocally moving handles and arm supports for use during training. The commercial harness was uncomfortable for one participant, despite adding padding. This challenge is common across locomotor training devices and warrants further attention to enable comfortable engagement in intensive locomotor exercise. 
The ICARE technology is currently being used in rehabilitation, medical fitness and home settings for adults and adolescents approaching at least $\sim 1.5$ meters in height. The "footprint" for the motor assisted elliptical, bodyweight support system, and adjustable seat is similar to that of a traditional elliptical. The device allows for use as a traditional elliptical in addition to the motor-assisted ICARE features. For those requiring additional assistance, supplemental stairs and ramps are available. Future research, exploring outcomes arising from use of the pediatric adapted ICARE in a community-based environment, is critical for guiding understanding of opportunities and limitations of the technology in a real-world environment.

\subsection{Gait}

The protocol enabled each child to achieve thou sands of repetitions of step-like movements as advocated by neuronal plasticity paradigms [2]. While there is a paucity of literature directly related to gait changes following locomotor interventions for children with chronic ABI [28], preliminary findings from the current feasibility study compare positively with improvements reported following locomotor training in other pediatric populations. Participants in the current study demonstrated increases in comfort- able walking speed post-intervention (Child $1=23 \%$, Child $2=82 \%$, and Child $3=10 \%$ ). Longer 2MWT distances were also documented following the 24 training sessions (Child $1=2 \%$, Child $2=$ $42 \%$, and Child $3=13 \%$ ). In contrast, following 20 Lokomat $\AA$ sessions delivered over four weeks in conjunction with inpatient rehabilitation, children with acute $\mathrm{ABI}$ demonstrated a 33\% increase in average comfort- able walking speed (from 0.49 to $0.65 \mathrm{~m} / \mathrm{s}$ ) [29] and a $30 \%$ longer 6-Minute Walk Test distance (from 233 to $304 \mathrm{~m}$ ) [30]. However, the Lokomat $\AA$ intervention was delivered as an adjunct to inpatient rehabilitation, thus impact cannot be isolated from the overall rehabilitation program's affects. Additionally, children receiving the Lokomat $®$ intervention were earlier in their recovery, thus increasing likelihood of spontaneous recovery contributing to gains [31]. In a separate study, children with cerebral palsy (CP) increased walking speed 25\% (from $0.87 \mathrm{~m} / \mathrm{s}$ to $1.09 \mathrm{~m} / \mathrm{s}$ ) following 12 robotic-assisted gait sessions [32]. However, most participants also received Botox injections that may have reduced lower extremity spasticity, potentially confounding interpretation of the robotic intervention's impact [32]. 
To our knowledge, the only study performed to date in a community-dwelling population of children with brain injury delivered a homebased exercise intervention for a small cohort with severe traumatic brain injury ( $n=5)$ and cerebral palsy ( $n=5$, Gross Motor Function Classification System levels I and II) [33]. Consistent with our findings, improvements in 2MWT (7\%, from 114.1 to 122.1 meters) and walking speed (4\%, from 0.96 to $1.0 \mathrm{~m} / \mathrm{s}$ ) were documented. In contrast to our findings, their documented differences did not surpass the clinical detectable thresholds observed with our participants.

While not assessed in the current feasibility study, it is reasonable to suspect that walking improvements may have enhanced children's capacity in community settings. Child 1's comfortable walking speed improved by $23 \%$ to $0.87 \mathrm{~m} / \mathrm{s}$, a speed exceeding teachers' perceptions of the slowest acceptable walking speed for second- and third-grade students with mobility challenges $(0.79 \mathrm{~m} / \mathrm{s})$ [34]. Child 2's and Child 3's comfortable walking speed following training $(1.67 \mathrm{~m} / \mathrm{s}$ and $1.34 \mathrm{~m} / \mathrm{s}$, respectively) easily surpassed the pace used by typically developing first through third grade peers who serve as "line leaders" during elementary school hallway activities (1.16 to $1.25 \mathrm{~m} / \mathrm{s}$ ) [34] as well as the speed needed to safely cross most U.S. street intersections (1.32 m/s) [35].

It is unknown whether the walking improvements arose solely from increased repetition of the gait-like movements across the 24 ICARE training sessions, or simply from engagement in some form of physical activity. While beyond the scope of this initial feasibility study, a future randomized controlled trial, comparing walking outcomes arising from ICARE training to those emerging from training at similar repetition/intensity levels on an alternative device could help discern the potential impact of task-related training on the ICARE vs. other forms of physical activity on gait improvements.

\subsection{Cardiorespiratory challenge}

Beyond encouraging mass repetition of stepping, the program also promoted sustained cardiorespiratory challenge. Current guidelines for school-aged children recommend daily engagement in 60 minutes of moderate- to vigorous-intensity exercise, with vigorous-intensity aerobic physical activity occur ring at least 3 days/week [6]. While no participant achieved these recommendations during early sessions, each 
displayed marked progress toward this goal across the program. Child 1 progressed from completing 51 minutes of moderate-intensity exercise during week one, to engaging in approximately 152 minutes of moderate- and vigorous-intensity exercise during her final week. Child 2 progressed from 68 minutes of moderate-intensity exercise during week one, to 176 minutes of moderate-intensity exercise during her final three sessions. We suspect her HR during ICARE training was blunted given use of an alpha-2A adrenergic receptor agonist. Child 2 experienced the greatest increase in the 2MWT (an indirect measure of fitness). Child 3 progressed from 64 minutes of moderate-intensity exercise and 2 minutes of vigorous-intensity exercise during her first week to 173 minutes of moderate- to vigorous intensity exercise.

Additionally, each child's capacity to engage in longer and more challenging sessions (i.e., faster speed and greater time overriding motorassistance) as the program progressed serves as a clinically meaningful measure of improved fitness as does the 11- to 19-point drop in resting HR from beginning to end of the program [36]. It is unknown how long fitness gains were sustained following program cessation and whether similar gains could be achieved if sessions were scheduled only 2 days/ week. Additionally, the current work did not characterize the nature of activities children performed on a day-to-day basis.

\subsection{Balance}

While the pattern of change in PBS static scores from pre- to post-intervention was inconsistent across the three children (Child $1=+7 \%$, Child $2=-7 \%$, and Child $3=0 \%$ ), each participant's PBS dynamic score consistently increased following training (Child $1=43 \%$, Child $2=18 \%$, and Child $3=3 \%$ ). Despite Child 1's improvement in the PBS dynamic score, she completed the mTUG, which has also been used to assess dynamic balance, more slowly post- intervention (2\% decrease in speed). In contrast, the mTUG was performed more rapidly post-intervention by Child 2 (9\%) and Child 3 (19\%). Child 3 greatly surpassed the mTUG MCID and achieved a similar time to age-matched typically developing children [5]. Given previous research demonstrating increased lower extremity muscle demands at faster ICARE speeds and when overriding motor-assistance $[10,15]$, it is possible that the current program's training progression improved lower extremity muscle strength critical 
for dynamic balance responses. Additionally, sustaining upright control of the head, arms and trunk over the dynamically moving base of support (i.e., pedals) may have also challenged vestibular and proprioceptive systems [37]. How- ever, musculoskeletal, vestibular, and proprioceptive changes were not formally assessed in this study, pre- venting understanding of whether potential changes in these systems contributed to balance improvements documented following training.

\section{Conclusion}

This feasibility study involving three children pro- vides preliminary data to suggest that an ICARE modified to address the needs of individuals of smaller stature can be used by children with ABI who have limited walking and fitness capacity. Each child achieved tens of thousands of repetitions of step-like movements over the 24-session intervention while simultaneously engaging in moderate- to vigorous- intensity physical activity. Although promising, the findings from this feasibility study must be interpreted with caution as it is unknown whether the documented improvements in the three children with ABI arose purely from the greater engagement in physical activity or from the combination of the training program with the novel ICARE technology. Additionally, the longer-term impact of this intervention on not only function, fitness, and balance, but also health, cognition, and emotional wellbeing has yet to be explored. A larger scale randomized controlled study comparing walking, fitness, and balance outcomes in children with ABI arising from a structured intervention delivered on the ICARE versus an alternative technology (e.g., recumbent cycle) could help elucidate the relative importance of each technology to improvements. If both interventions lead to similar and significant improvements in function, fitness and balance, then critical factors such as child/clinician preference, technology expense, and space constraints could be used to guide technology selection in various community environments. Future research should also explore the extent to which laboratory-based improvements are repeatable and translatable into real-world activities. Addition ally, systematic evaluation of strength, vestibular and proprioceptive capacity before and following the intervention could help advance understanding of underlying factors that might contribute to improved walking, fitness and balance. 
Acknowledgments We would like to acknowledge Sonya Irons, PT, DPT, CCS, Alex Garbin, DPT, and Nicole Schwery, MS, CSCS, for their assistance with the children's training and clinical assessments.

Conflict of interest The prototype technology that was evaluated in the current manuscript was adapted from a patented motor-assisted elliptical technology created for adults by Judith M. Burnfield and Thad W. Buster through a grant previously received from the National Institute on Disability and Rehabilitation Research, Department of Education (H133G070209; Principal Investigator: Burnfield). The adult technology has been licensed to Sports Art for commercial distribution. Madonna Rehabilitation Hospitals receives royalties and a portion of these royalties is shared with inventors Judith $M$. Burnfield and Thad W. Buster. Guilherme M. Cesar has no conflict of interest to report.

Funding The contents of this work were developed under a grant initially received from the National Institute on Disability and Rehabilitation Research, Department of Education (H133G130274; Principal Investigator: Burnfield) and subsequently funded through a grant from the National Institute on Disability, Independent Living, and Rehabilitation Research, Administration for Community Living (90IF0060; Principal Investigator: Burnfield). The contents of the article do not necessarily represent the policy of the Department of Education or the Administration for Community Living, and endorsement by the federal government should not be assumed.

\section{References}

[1] Seif-Naraghi AH, Herman RM. A novel method for locomotion training. J Head Trauma Rehabil. 1999;14(2):146-62. doi: 10.1097/00001199-199904000-00005.

[2] Lang CE, Macdonald JR, Reisman DS, Boyd L, Jacob- son Kimberly T, SchindlerIvens SM, et al. Observation of amounts of movement practice provided during stroke rehabilitation. Arch Phys Med Rehabil. 2009;90(10):1692-8. doi: 10.1016/j.apmr.2009.04.005.

[3] United States Government Accountability Office. Students with disabilities: More information and guidance could improve opportunities in physical education and athletics. 2010. Available from: http://www.gao.gov/new.items/d10519. pdf

[4] Rossi C, Sullivan J. Motor fitness in children and adolescents with traumatic brain injury. Arch Phys Med Rehabil. 1996;77:1062-5. doi: 10.1016/ s0003-9993(96)90069-6.

[5] Katz-Leurer M, Rotem H, Keren O, Meyer S. Balance abilities and gait characteristics in post-traumatic brain injury, cerebral palsy and typically developed children. Dev Neurorehabil. 2009;12(2):100-5. doi: 10.1080/175184209028 00928.

[6] U.S. Department of Health and Human Services. Physical Activity Guidelines for Americans, 2nd edition. Washington, DC. 2018. 
[7] Devine JM, Zafonte RD. Physical exercise and cognitive recovery in acquired brain injury: a review of the literature. PM R. 2009;1(6):560-75. doi: 10.1016/j. pmrj.2009.03.015.

[8] Burnfield JM, Buster TW, Pfeifer CM, Irons SL, Cesar GM, Nelson CA. Adapted motor-assisted elliptical for rehabilitation of children with physical disabilities. J Med Devices. 2019;13(1):011006. doi: 10.1115/1.4041588.

[9] Burnfield J, Pfeifer C, Kwapiszeski S, Irons S, Buster T, Cesar G. Impact of ICARE training speed and motor assistance on cardiovascular response. Cardiopul Phys Ther J. 2019;30(3):115-22. doi: doi: 10.1097/CPT.00000000000 00098.

[10] Burnfield JM, Cesar GM, Buster TW, Irons SL, Nelson CA. Kinematic and muscle demand similarities between motor- assisted elliptical training and walking: Implications for pediatric gait rehabilitation. Gait Posture. 2017;51:194-200. doi: 10.1016/j.gaitpost.2016.10.018.

[11] Burnfield JM, Cesar GM, Buster TW, Irons SL, Pfeifer CM. Walking and fitness improvements in child with diplegic cerebral palsy following motor-assisted elliptical intervention. Ped Phys Ther. 2018;30(4):E1-E7. doi: 10.1097/ PEP.0000000000000541.

[12] Cesar GM, Buster TW, Burnfield JM. Cardiorespiratory fit ness, balance and walking improvements in an adolescent with cerebral palsy (GMFCS II) and autism after motor assisted elliptical training. Eur J Physiother. 2020;22(3): 124-32. doi: 10.1080/21679169.2018.1536764.

[13] Irons SL, Brusola GA, Buster TW, Burnfield JM. Novel motor-assisted elliptical training intervention improves Six- Minute Walk Test and oxygen cost for an individual with progressive supranuclear palsy. Cardiopulm Phys Ther J. 2015;26(2):36-41. doi: 10.1097/CPT.0000000000000027

[14] McCrory B, Harlow AH, Burnfield JM. Musculoskeletal risk to physical therapists during overground gait training: A case report. Proceedings of the Human Factors and Ergonomics Society Annual Meeting. 2014;58(1):1219-23. doi: 10.1177/1541931214581254.

[15] Burnfield JM, Irons SL, Buster TW, Taylor AP, Hildner GA, Shu Y. Comparative analysis of speed's impact on muscle demands during partial body weight support motor-assisted elliptical training. Gait Posture. 2014;39(1):314-20. doi: 10.1016/j.gaitpost.2013.07.120.

[16] Machado FA, Denadai BS. Validity of maximum heart rate prediction equations for children and adolescents. Arq Bras Cardiol. 2011;97(2):136-40. doi: 10.1590/s0066-782x2011005000078.

[17] Macko RF. Chapter 25: Stroke, Brain Trauma, and Spinal Cord Injuries. In: Moore GE, Durstine JL, Painter PL, editors. ACSM's exercise management for persons with chronic diseases and disabilities. Champaign, IL: Human Kinetics; 2016.

[18] Pfeifer CM, Rowen DA, Buster TW, Cesar GM, Irons SL, Burnfield JM. Design and validation of a heart rate and speed monitoring device with Intelligently Con trolled Assistive Rehabilitation Elliptical. J Med Devices. 2019;13(1):015002.

[19] Bohannon RW, Bubela D, Magasi S, McCreath H, Wang YC, Reuben D, et al. Comparison of walking performance over the first 2 minutes and the full 
6 minutes of the Six-Minute Walk Test. BMC Res Notes. 2014;7:269. doi: 10.1186/1756-0500-7-269.

[20] Williams EN, Carroll SG, Reddihough DS, Phillips BA, Galea MP. Investigation of the timed 'Up \& Go' test in children. Dev Med Child Neurol. 2005;47(8):518-24. doi: $10.1017 / \mathrm{s} 0012162205001027$.

[21] Franjoine MR, Gunther JS, Taylor MJ. Pediatric balance scale: A modified version of the Berg Balance Scale for the school-age child with mild to moderate motor impairment. Pediatr Phys Ther. 2003;15(2):114-28. doi: 10.1097/01. PEP.0000068117.48023.18

[22] Faul F, Erdfelder E, Lang AG, Buchner A. G* Power 3: a flexible statistical power analysis program for the social, behavioral, and biomedical sciences. Behav Res Methods. 2007;39(2):175-91. doi: 10.3758/bf03193146

[23] Faul F, Erdfelder E, Buchner A, Lang AG. Statistical power analyses using G* Power 3.1: Tests for correlation and regression analyses. Behav Res Methods. 2009;41(4):1149-60. doi: 10.3758/BRM.41.4.1149

[24] Chen CL, Shen IH, Chen CY, Wu CY, Liu WY, Chung CY. Validity, responsiveness, minimal detectable change, and minimal clinically important change of Pediatric Balance Scale in children with cerebral palsy. Res Dev Disabil. 2013;34(3):916-22. doi: 10.1016/j.ridd.2012.11.006.

[25] Pin TW, Choi HL. Reliability, validity, and norms of the 2- min walk test in children with and without neuromuscular disorders aged 6-12. Disabil Rehabil. 2018;40(11):1266-72. doi: 10.1080/09638288.2017.1294208.

[26] Page P. Beyond statistical significance: clinical interpretation of rehabilitation research literature. Int J Sports Phys Ther. 2014;9(5):726-36.

[27] Burnfield JM, Buster TW, Goldman AJ, Corbridge LM, Harper-Hanigan K. Partial body weight support treadmill training speed influences paretic and nonparetic leg muscle activation, stride characteristics, and ratings of perceived exertion during acute stroke rehabilitation. Hum Mov Sci. 2016;47:16-28. doi: 10.1016/j.humov.2016.01.012

[28] Lefmann S, Russo R, Hillier S. The effectiveness of robotic-assisted gait training for paediatric gait disorders: systematic review. J Neuroeng Rehabil. 2017;14(1):1. doi: 10.1186/s12984-016-0214-x.

[29] Beretta E, Romei M, Molteni E, Avantaggiato P, Strazzer S. Combined roboticaided gait training and physical therapy improve functional abilities and hip kinematics during gait in children and adolescents with acquired brain injury. Brain Inj. 2015;29(7-8):955-62. doi: 10.3109/02699052. 2015.1005130.

[30] Beretta E, Storm FA, Strazzer S, Frascarelli F, Petrarca M, Colazza A, et al. Effect of robot-assisted gait training in a large population of children with motor impairment due to cerebral palsy or acquired brain injury. Arch Phys Med Rehabil. 2019;101(1):106-12. doi: 10.1016/j.apmr.2019.08.479.

[31] Bennett CN, Gupta RK, Prabhakar P, Christopher R, Sampath S, Thennarasu K, et al. Clinical and biochemical outcomes following EEG neurofeedback training in traumatic brain injury in the context of spontaneous recovery. Clin EEG Neurosci. 2018;49(6):433-40. doi: 10.1177/ 1550059417744899. 
[32] Meyer-Heim A, Borggraefe I, Amman-Reiffer C, Berweck S, Sennhauser FH, Colombo G, et al. Feasibility of robotic- assisted locomotor training in children with central gait impairment. Dev Med Child Neurol. 2007;49:900-6. doi: 10.1111/j.1469-8749.2007.00900.x.

[33] Katz-Leurer M, Rotem H, Keren O, Meyer S. The effects of a 'home-based' taskoriented exercise programme on motor and balance performance in children with spastic cerebral palsy and severe traumatic brain injury. Clin Rehabil. 2009;23(8):714-24. doi: 10.1177/0269215509335293.

[34] David KS, Sullivan M. Expectations for walking speeds: Standards for students in elementary schools. Pediatr Phys Ther. 2005;17(2):120-7. doi: 10.1097/01. pep.0000163074. 89545.fa.

[35] Salbach NM, O'Brien K, Brooks D, Irvin E, Martino R, Takhar P, et al. Speed and distance requirements for com- munity ambulation: a systematic review. Arch Phys Med Rehabil. 2014;95(1):117-28 e11. doi: 10.1016/j.apmr.2013. 06.017.

[36] Silva DAS, de Lima TR, Tremblay MS. Association between resting heart rate and health-related physical fitness in Brazilian adolescents. Biomed Res Int. 2018;2018: 3812197. doi: 10.1155/2018/3812197.

[37] Schieppati M, Giordano A, Nardone A. Variability in a dynamic postural task attests ample flexibility in balance control mechanisms. Exp Brain Res.

2002;144(2):200-10. doi: 10.1007/s00221-002-1028-6. 